\title{
Verzeichnis der in den Briefen erwähnten Schriften von Johann Christoph Gottsched und Luise Adelgunde Victorie Gottsched
}

\author{
Johann Christoph Gottsched
}

Geordnet nach Phillip Marshall Mitchell: Gottsched-Bibliographie (Johann Christoph Gottsched: Ausgewählte Werke 12). Berlin 1987.

\section{1}

Gottsched zugeschrieben: Den Herren T. S. beehrte als einen wehrten Freund und Wohlthäter J. C. G. In: Menantes [Christian Friedrich Hunold]: Auserlesene und theils noch nie gedruckte Gedichte unterschiedener Berühmten und geschickten Männer. 26. Stück. Halle: Neue Buchhandlung, 1721, S. 570-574: S. 121

\section{5}

Herrn D. Johann Valentin Pietschen ... Gesamlete Poetische Nr. 28 Schriften: S. 395

\section{6}

Die Vernünftigen Tadlerinnen Erster Jahr-Theil: S. 120, 410 Nr. 29

\section{7}

Die Vernünftigen Tadlerinnen Andrer Jahr-Theil: S. 120, $410 \quad$ Nr. 35

Nachricht von der erneuerten Deutschen Gesellschafft in Nr. 36 Leipzig: S. 343

\section{8}

Der Biedermann Erster Theil: S. 120, 410

Nr. 39

Oden Der Deutschen Gesellschaft in Leipzig: S. 203, 343

Nr. 42

Grundriß Zu einer Vernünfftmäßigen Redekunst: S. 111

Nr. 72, hier unter 1729 verzeichnet, obwohl 1728 erschienen 
enthält:

S. 205-229:

Marcus Tullius Cicero: Vertheidigungs=Rede vor den Poeten

Nicht bei Mitchell

A. Licin. Archias: S. 341

S. 257-309:

Lob=Rede Auf den Königl. Französischen General=Feld=Mar-

Nicht bei Mitchell schall, Grafen von Turenne, gehalten zu Paris in der Kirche des Heil. Eustachius, Den 10. Jenn. 1676. durch Esprit Fleschier: S. 180

\section{9}

Der Biedermann Zweyter Theil: S. 120, 410, 417

Nr. 70

Grundriß Zu einer Vernünfftmäßigen Redekunst

Nr. 72, s. 1728

Versuch einer Critischen Dichtkunst vor die Deutschen:

Nr. 75

S. $111,120,131,336$

\section{0}

Der Deutschen Gesellschaft in Leipzig Eigene Schriften und Ubersetzungen: S. 203, 343

Lucians Abbildung einer vollkommenen Schönheit aus dem Griechischen übersetzt, Und der schönen und geistreichen L. A. V. K. an ihrem Geburtstage 1730. den 11. April zugeeignet von Johann Christoph Gottscheden. In: Deutsche Gesellschaft, Eigene Schriften 2, 1734,

S. 461-480: S. 377

\section{1}

Nachricht von der Deutschen Gesellschaft zu Leipzig: S. 189, 341,343

\section{2}

Sterbender Cato: S. 39, 49, 247-249, 312, 314, 403

Nr. 92

Der Deutschen Gesellschaft in Leipzig Gesammlete Reden und Nr. 94 Gedichte: S. 343

Beyträge zur Critischen Historie der deutschen Sprache, Poesie und Beredsamkeit. 1.-4. Stück (1732-1733): S. 12, 13, 89, 178, 203, 206, 293, 294, 392

Als Se. Magnificenz, Herr D. Carl Otto Rechenberg, im October 1732. die Aufsicht in dem Königl. und Churfürstl. Convictorio zu Leipzig übernommen hatten. In: Gottsched, Gedichte, 1736, S. 412-415: S. 416

Nr. 80

s. 1734 , Nr. 141 
Critische Anmerkungen über D. C. von Lohenstein Lobrede

Nicht bei Mitchell bey ... Christians von Hofmannswaldau ... Leichenbegängnisse gehalten. In: Beiträge 1/3 (1732), S. 496-526: S. 12

\section{3}

Ein wahrer Held und Vollkommener Regent: S. 110, 333, 334 Nr. 111

$\begin{array}{ll}\text { Sachsens völlig ersetzter Verlust: S. 110, } 111 & \text { Nr. } 112\end{array}$

Erste Gründe Der Gesamten Weltweisheit. Theoretischer Nr. 114

Theil: S. 51, 60, 111, 147, 177, 202, 211, 279, 447

Daß die Poesie am geschicktesten sey: S. 34, $35 \quad$ Nr. 117

Beyträge zur Critischen Historie der deutschen Sprache, Poesie Nr. 123 und Beredsamkeit. 5.-8. Stück (1733-1734): S. 4, 49, 65, 69, 124, 130-132, 191, 203, 370

Herrn Heinrich Anshelm von Ziegler und Kliphausen Asiatische Banise ... Leipzig bey Thomas Fritschen. 1688. In: Beiträge 2/6 (1733), S. 274-292: S. 13

Bescheidene Antwort auf die vorhergehenden Critischen Gedanken über den sterbenden Cato. In: Beiträge 2/5 (1733), S. 44-68: S. 4, 49

Atalanta oder Die bezwungene Sprödigkeit: S. 49, 195, 249

Ueber des Herrn Hofpredigers Colers Paßionsbetrachtungen. In: Gottsched, Gedichte, 1751, 1, S. 148-151: S. 26

\section{4}

Erste Gründe Der Gesamten Weltweisheit. Andrer Practischer Theil: S. 23, 40, 51, 55, 110, 111, 135, 146, 147, 174, $177,202,211,277,279,447$

Iniquitatem exterorum in ferendo in eruditis nostratibus iudicio ... sistit: S. $41,44,56,57,85,87,98$

Oratio pro utilitate et necessitate metaphysicae: S. 40, 48, 56, $57,62,79,85,202$

Der Deutschen Gesellschaft in Leipzig Eigene Schriften und Ubersetzungen. Der Andere Theil: S. 55, 145, 169, 170, $171,193,203,235,246,269,275,343,417$

enthält:

S. 461-480:

Nr. 141, s. 1730

Lucians Abbildung einer vollkommenen Schönheit

s. $1741, \mathrm{Nr} .232$ und 1742, Nr. 244

Nicht bei Mitchell

Nr. 129

Nr. 130

Nr. 132 
S. 565-570:

Probe einer Uebersetzung aus Ramsays reisendem Cyrus:

Nr. 142

S. 113,128

S. 684-687

Schreiben des berühmten griechischen Poeten Menanders an seine geliebte Glycera: S. 244

Nr. 143

S. 687-692:

Glycera an den Menander: S. 244

Nicht bei Mitchell

Des Herrn Racine Trauerspiel Iphigenia: S. 35, 49, 176, 249 , 403

Beyträge zur Critischen Historie der deutschen Sprache, Poesie und Beredsamkeit. 9.-12. Stück (1734-1735): S. 64, 65, 173, 174, 177-179, 183, 190, 192, 193, 197, 203, 205, $254,259,278,285,294,306,307,326,332,358,360$

Ludwig Friedrich Hudemanns Probe einiger Gedichte und Poetischen Ubersetzungen ... In: Beiträge 3/10 (1734), S. 268-316: S. 336,338

Christian Thomasens kleine deutsche Schriften, mit Fleiß zusammen getragen, und zum andernmale gedruckt. Halle 1707. In: Beiträge 3/10 (1734), S. 348-358: S. 190, 191

An den ... Herzog zu Braunschweig und Lüneburg etc. etc. als Derselbe im 1734 Jahre in dem Lauchstädter Bade, bey der Tafel die Gnade gehabt hatte, dem Verfasser auf den Flor des deutschen Parnasses zuzutrinken. In: Gottsched, Gedichte, 1751, 1, S. 67-69: S. 144, 162

Anhang zur natürlichen Gottesgelahrheit. Philosophisches Gespräche, über die Frage: $\mathrm{Ob}$ mehr als ein unendliches Wesen seyn könne?: S. 147

An Jungfer L. A. V. Kulmus. 1734, den 11 April. In: AW 1, S. 390-394: S. 152 Nicht bei Mitchell

Auf Sr. Wohlerwürden, Herrn M. Pantkens, Trauungsceremonie. In: Gottsched, Gedichte, 1736, S. 422 f. und Gedichte, 1751, 1, S. 350 f.: S. 58, 59, 108, 109

Dankgedicht, an die unvergleichliche Victoria, für Dero überschicktes Bildniß. 1734. um Neujahr. In: L. A. V. Gottsched, Kleinere Gedichte, S. 199-203: S. 46

Nicht bei Mitchell

Nicht bei Mitchell

Nicht bei Mitchell

Nicht bei Mitchell 
Den höchstpreiswürdigen Doctorhut, Welchen Der ... Herr

Nicht bei Mitchell

Franz Ludewig ... Graf zu Oettingen ... Im Jahr 1734 den 12 May auf der hochberühmten Universität Helmstädt ... erhielte ... Leipzig: Bernhard Christoph Breitkopf,

1734: S. 95, 146, 212, 333

Urania, ein Schäfergedicht 1734. In: L.A. V. Gottsched,

Kleinere Gedichte, S. 206-212: S. 62, 104, 106, 152

Nicht bei Mitchell

\section{5}

Sterbender Cato. Andere Auflage: S. $424 \quad$ Nr. 150

Beyträge zur Critischen Historie der deutschen Sprache, Poesie Nr. 165 und Beredsamkeit. 13.-16. Stück (1735-1737): S. 138, $360,378,393,394,397,409,453$

\section{6}

Gedichte: S. 446

Nr. 171

Ausführliche Redekunst: S. 111, 298

Nr. 174

\section{8}

Beyträge zur Critischen Historie der deutschen Sprache, Poesie

Nr. 180 und Beredsamkeit. 13.-16. Stück (1735-1737): S. 333, $334,420-427$

\section{9}

Beyträge zur Critischen Historie der deutschen Sprache, Poesie Nr. 217 und Beredsamkeit. 21.-24. Stück (1739-1740): S. 203

\section{1}

Atalanta oder Die bezwungene Sprödigkeit. In: Die Deutsche Schaubühne ... Dritter Theil. Leipzig: Bernhard Christoph Breitkopf, 1741, S. 366-442 Nr. 232, s. 1733 und 1742, Nr. 244

\section{2}

Atalanta oder Die bezwungene Sprödigkeit. Hamburg: Johann Diderich Nottebohm, 1742

Nr. 232, s. 1733 und 1742, Nr. 244

Nr. 244, s. 1733 und 1741, Nr. 232

\section{Unveröffentlichte Werke}

Ausgabe der Werke von Martin Opitz: S. 20, 55, 86, 135, 136, 279, 451 
Luise Adelgunde Victorie Gottsched

1731

Lambert, Anne-Thérèse de: Neue Betrachtungen über das Frauenzimmer, aus dem Französischen übersetzt durch ein junges Frauenzimmer aus ${ }^{* * *}$ und herausgegeben von einem Mitgliede der Deutschen Gesellschaft in Leipzig. Leipzig: Bernhard Christoph Breitkopf, 1731: S. 292, 418

enthält S. 49-78:

Der Ubersetzerin Eigene Gedichte: S. 292

\section{4}

Aus dem Sammler (Glaneur) vom Jahr 1733. den 26. März. Vergleichung des Theophrasts und des Hrn. v. la Bruyere. In: Runckel 1, S. 191-197; Ueber den Nutzen der Schauspiele. In: Runckel 1, S. 197-207: S. 296

Horatius Flaccus, Quintus: Oden: S. 155, 296

\section{5}

Addison, Joseph: Cato, Ein Trauerspiel, Aus dem Englischen ... übersetzt von Luise Adelg. Victoria Gottsched, geb. Kulmus. Leipzig: Bernhard Christoph Breitkopf, 1735: S. $273,296,390,425$

Gomez, Madeleine-Angélique Poisson de: Der Sieg der Beredsamkeit ... übersetzt, durch Luise Adelg. Victoria Kulmus. Leipzig: Bernhard Christoph Breitkopf, 1735: S. 53, 267, 273, 292, 328, 359, 377, 417

\section{Unveröffentlichte Werke}

Übersetzung (vor 1731) von Marie Madeleine Pioche de LaVergne de LaFayette: La Princesse de Clèves. Paris 1678 u. ö.: S. 328 